\title{
Determining the Acetic Acid Concentration in White Vinegar: An At-Home Undergraduate Chemistry Experiment During the COVID-19 Pandemic
}

\author{
Philip Parel $^{1 \dagger}$, Lydia Burnett ${ }^{1 \dagger}$, Morgan Geoffroy ${ }^{1 \dagger}$, John Parel $^{2}$, Ling Hao ${ }^{1 *}$ \\ ${ }^{1}$ Department of Chemistry, The George Washington University, 800 22nd Street NW, Washington, D.C. 20052, USA. \\ ${ }^{2}$ Suncoast Community High School, 1717 Avenue S, Riviera Beach, FL 33404, USA
}

\begin{abstract}
Due to the COVID-19 pandemic, many university-level chemistry laboratory experiments transitioned online. This created an educational challenge because students are unable to access reagents and instruments typical in a university lab setting. It is also difficult for students to connect theory to practice since real-lab experimentation is not possible in a virtual online format. In our online Introductory Quantitative Analysis Laboratory course (GW Chem 2123W) in spring 2021, students were required to design and perform an experiment that could be safely conducted at home which can also demonstrate key principles of quantitative analysis. Herein, we conducted a reliable at-home experiment that utilizes university-level acid/base titration techniques to determine the acetic acid concentration in white vinegar. The experiment used sodium bicarbonate in the form of baking soda to titrate the Whole Foods white vinegar which is advertised to contain $6 \%$ of acetic acid. We reliably obtained an equivalence point for the titration from which the actual concentration of acetic acid in the vinegar was calculated with error analysis to be $19 \%$ higher than the advertised value. The analytical techniques demonstrated in this experiment supplement practical knowledge of acid/base techniques learned in the online classroom and prompt adoption of this methodology in online laboratory curriculum. This experiment can also be readily applied to measure other acidic solutions such as vinegar and juice for at-home experiment.
\end{abstract}

KEYWORDS: At-Home Experiment, COVID-19, Pandemic, Titration, White Vinegar, Acetic Acid

\section{INTRODUCTION:}

\section{At-Home Experiment During the Pandemic}

During the outbreak of the COVID-19 pandemic, majority of higher education institutions transitioned to online learning environment. ${ }^{1,2}$ However for virtual laboratory courses, students face educational challenges in connecting the theoretical concepts learned in class to practical applications in a real laboratory setting. ${ }^{3}$ One possible solution is to conduct modified versions of laboratory experiments at home, so called "kitchen chemistry". ${ }^{4}$ But experimental safety issues need to carefully investigated for at-home experiments. Schultz et al. ${ }^{4}$ found that students can complete at-home experiments at similar rate compared to real-lab experiment to achieve the desired learning outcomes with similar mean scores of post-lab assignment..$^{5}$ Moreover, Andrews et al. ${ }^{1}$ have provided a series examples of at-home lab experiments, ranging from concepts in acid-base equilibria to thermodynamics. Here, we modified an acid-base titration experiment to demonstrate how university-level labs can be conducted safely at-home. Further, acetic acid is a ubiquitous household ingredient in vinegar known to promote health complications, including increased inflammation and skin irritation in high concentrations. ${ }^{6,7}$ Thus, we are interested in verifying the acetic acid concentration in Whole Foods brand white wine vinegar to both address this health issue and to propose an at-home methodology that can be used to address the educational gap created by online laboratory courses.
Previous titration experimentations for acetic acid content have largely been dependent on university-level apparatus, but few experiments have been adapted to test the acetic acid concentration in vinegar at home. ${ }^{1,8,9}$ During our Chem $2123 \mathrm{~W}$ course, we first used ChemCollective webiste (http://chemcollective.org) to learn the theoretical knowledge, practice titrations and applied what we learned to design our own experiment. ${ }^{9}$ While the ChemCollective titration was useful for at-home learning, it still lacks the benefits of performing and monitoring an experiment in-person as would be done in a real laboratory. We design a procedure that can fill this disparity by expanding on a previous experiment proposed by Andrews et al. ${ }^{1}$ We proposed an accurate and replicable methodology that still employs higher-level analytical techniques conducted during university-level experiments, such as error analysis and the determination of percent composition through titration. Yet, our method is more accessible to pandemic-era students who only have access to common household goods and may not have access to the imaging subscriptions, such as Adobe Photoshop, used by Andrews et al. ${ }^{1}$ Our methodology has grounds to be incorporated into the online curriculum of university and high school chemistry laboratory courses because it provides a practical, at-home approach to acid/base titrations while still being rigorous enough to educate students on titration chemistry and educating them on an experiment that is significant to human health. Our procedure also provides a reliable method through which the acetic acid content of vinegar products can be verified. 


\section{Rationale of Analyzing White Vinegar}

Standard white vinegar is a solution that generally contains $4 \%-7 \%$ acetic acid by weight in approximately $93 \%-96 \%$ distilled water. ${ }^{6}$ Acetic acid is a common byproduct of fermentation of carbohydrates and it has been used historically for a wide variety of medicinal purposes, including wound treatment, cures for stomachaches, and even the treatment of diabetes before modern treatments were available. ${ }^{6}$ Vinegar has been shown to aid in weight loss when consumed with a meal by increasing feelings of satiety. ${ }^{10}$

Despite its widespread use, acetic acid has been reported to have several harmful effects associated with its acidity, specifically in the form of esophageal inflammation. ${ }^{6}$ Prior reports demonstrate patients who experienced oropharyngeal inflammation and second-degree erosive injury of the esophagus upon ingestion of minute amounts of vinegar. $^{6}$ This pro-inflammatory effect of acetic acid in vinegar is of special interest because chronic consumption of vinegar has previously resulted in ulcers and mucosal bleeding along the length of the esophagus. ${ }^{6,11}$ Moreover, acetic acid is known to be a strong eye and skin irritant with sufficient contact resulting in rashes and the formation of thick, callous skin. ${ }^{7}$ Acetic acid also has a special affinity for the mucous membrane, which makes it extremely destructive to the upper respiratory tract if exposed. ${ }^{7}$

Further, vinegar is used as an alternative medicine treatment in some cultures to treat airway obstructions. ${ }^{6}$ Vinegar is often touted as a "cure-for-all" in folklore with usages ranging from treatment of urinary tract infections to constipation. $^{12,13}$ Thus, determination of the acetic acid content in commercial vinegar becomes further imperative considering these widespread cultural implications of using vinegar and the potentially harmful effects associated with elevated concentrations of the ingredient. Especially during the COVID-19 era in which home-based cooking has increased, it is important to be able to quantify and verify the percent composition of acetic acid in store-bought vinegar solutions for safety purposes. ${ }^{14}$ In this study, Whole Foods' 365 brand white vinegar, which advertises a $6 \%$ acidity by weight in a $500 \mathrm{~mL}$ glass container, was selected as a proof-of-principle sample.

\section{Chemical Theory of the Acid-Base Titration}

An Acid can be titrated with a base in order to completely dissociate the protons. But acetic acid is a weak acid and does not dissociate entirely in water. In a university laboratory, a strong base such as $\mathrm{NaOH}$ would have been used to carry out the titration of acetic acid to completely dissociate protons from the acid. However, a strong base like $\mathrm{NaOH}$ would not be safe to work with outside of a laboratory. A safer alternative is $\mathrm{NaHCO}_{3}$, a weak base that is available at approximately $100 \%$ purity in the form of baking soda. ${ }^{15}$ The chemical reaction between sodium bicarbonate and acetic acid is shown below: ${ }^{16,17}$

$$
\begin{gathered}
\mathrm{CH}_{3} \mathrm{COOH}_{(a q)}+\mathrm{NaHCO}_{3(a q)} \rightleftharpoons \\
\mathrm{H}_{2} \mathrm{CO}_{3(a q)}+\mathrm{CH}_{3} \mathrm{COONa}_{(a q)} \text { (1) }
\end{gathered}
$$

Despite being a weak base, the reaction between bicarbonate and acetic acid produces carbonic acid, which decomposes into carbon dioxide and water in the following reactions: ${ }^{17}$

$$
\begin{gathered}
\mathrm{H}_{2} \mathrm{CO}_{3(a q)} \leftrightarrow \mathrm{H}_{2} \mathrm{O}_{(l)}+\mathrm{CO}_{2(a q)} \\
\mathrm{CO}_{2(a q)} \leftrightarrow \mathrm{CO}_{2(g)}
\end{gathered}
$$

The conversion of aqueous carbon dioxide to gaseous carbon dioxide is an endothermic reaction, so a weak acid with strong base neutralization can be simulated by adding heat to the reaction in Eq. $1 .{ }^{1}$ The addition of heat will promote the conversion of aqueous carbon dioxide to gaseous carbon dioxide in Eq. 3, thereby shifting the neutralization equilibrium nearly entirely to the right in accordance with Le Chatelier's principle and, thus, allowing a university-level weak acid/strong base titration to be conducted with more accessible household materials. ${ }^{1}$ By recording the $\mathrm{pH}$ as base is added, a titration curve can be created for acetic acid, from which the endpoint of the titration can be calculated by examining the point at which $\frac{d^{2}(p H)}{d V^{2}}=0 .{ }^{18}$ The concentration of acetic acid in the original vinegar analyte solution can be calculated using the concentration and volume of bicarbonate required to reach the endpoint of the titration. ${ }^{19}$ This calculated concentration of acetic acid can then be compared to the advertised value, which is approximately $1.0437 \mathrm{M}$, assuming that the density of the acetic acid solution is $1.0446 \mathrm{~g} / \mathrm{mL}$ at $25^{\circ} \mathrm{C}^{20}$ Precision is essential as there are limitations in the accuracy of household instruments.

\section{EXPERIMENTAL PROCEDURES}

\section{Materials}

3 teaspoons of $\sim 100 \% \mathrm{NaHCO}_{3}$ (Brand: Arm and Hammer Baking Soda), $300 \mathrm{~mL}$ of white vinegar ( $6 \%$ acidity, 365 by Whole Foods Market 365 Brand), $0.5 \mathrm{~mL}$ plastic syringe, two $100 \mathrm{~mL}$ measuring cups, two $500 \mathrm{~mL}$ measuring cup, digital $\mathrm{pH}$ meter (Brand: Ruolan, UNSPSC Code: 55111500), thermometer, $2 \mathrm{~L}$ of distilled $\mathrm{H}_{2} \mathrm{O}$, one electrical stove, one $3 \mathrm{~L}$ saucepan, 1 teaspoon, and 1 heat-safe cup to perform the reaction in. All materials were purchased from Whole Foods and Amazon.com and can be seen in Fig. 1A.

\section{Calibration of the pH Meter}

Prior to each trial, three calibration powders provided by the manufacturer were used to produce three solutions of known $\mathrm{pH}$ at 4.00, 6.86, and 9.18. Each powder was diluted and sufficiently mixed in $250 \mathrm{~mL}$ of distilled $\mathrm{H}_{2} \mathrm{O}$ and the Ruolan $\mathrm{pH}$ meter was calibrated in each solution to $\pm 0.01 \mathrm{pH}$, per manufacturer instructions. 


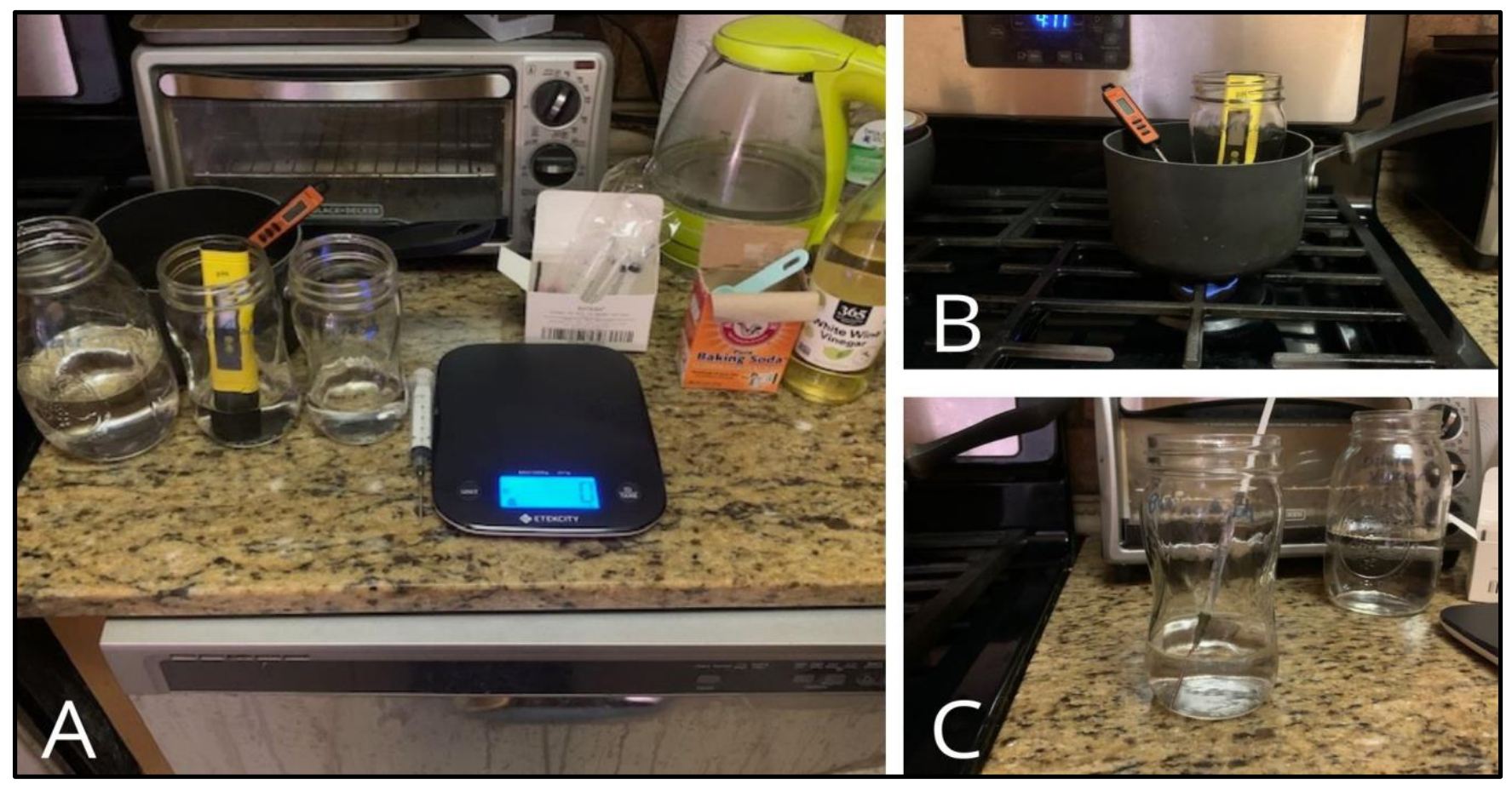

Figure 1 Experimental material and setup in the kitchen. A: Experimental materials including distilled water, mason jars (used for preparation of reagents and as reaction vessel), pot, thermometer, $\mathrm{pH}$ meter, medical syringes, kitchen scale, teaspoon, baking soda, white wine vinegar from Whole Foods. B: Reaction vessel containing diluted vinegar sits in a warm water bath, where the temperature is monitored using a digital kitchen thermometer. $\mathrm{pH}$ meter is inserted in the reaction vessel. In this image, the reaction vessel is labeled using a dry erase marker (optional). C: Baking soda solution in a separate container from the reaction vessel (labeled with a dry erase marker, optional). A $1 \mathrm{~mL}$ medical syringe is used to measure $0.5 \mathrm{~mL}$ aliquots of baking soda solution to add to the reaction vessel and titrate the vinegar.

The use of natural indicator from red cabbage extract can also be used to monitor the titration using colorimetry since the anthocyanin pigment of red cabbage ranges in the absorption of visible light from $\mathrm{pH} 2$ to $\mathrm{pH} 12 .{ }^{1}$ Previous studies used computer software, such as Photoshop, to analyze the red-green-blue composition of the titration solution at various intervals, making the indicator a suitable and easily accessible measure of the $\mathrm{pH}$ of the vinegar titration accurate to $0.3 \mathrm{pH} .^{1}$ However, due to the expensive software subscription for Adobe Photoshop and the better accuracy and conveniency of the Ruolan $\mathrm{pH}$ meter, we selected the $\mathrm{pH}$ meter for this at-home experiment.

\section{Preparation of the Dilute Vinegar Analyte Solution}

The vinegar solution was diluted by a factor of five by three students in the same group, each student completing one trial at home. In each trial, a $500 \mathrm{~mL}$ measuring cup was filled with $100 \mathrm{~mL}$ of white vinegar, then distilled $\mathrm{H}_{2} \mathrm{O}$ was added and sufficiently mixed until the final volume of the solution was $500 \mathrm{~mL}$. Then, $100 \mathrm{~mL}$ of this dilute solution was transferred to another $500 \mathrm{~mL}$ measuring cup to be used as the analyte solution. The average initial $\mathrm{pH}$ of this solution across three trials was 2.82 .

All measurements can also be accomplished using a kitchen scale instead of measuring cups. If students assume the density of both vinegar and distilled water are $1 \mathrm{~g} / \mathrm{mL}, 100 \mathrm{~mL}$ can be measured by weighing 100 grams of vinegar in a container which has been tared on the scale. 400 grams of water can be measured to achieve $400 \mathrm{~mL}$, and the vinegar can be added to water. However, students should be aware that the density of vinegar is not exactly $1 \mathrm{~g} / \mathrm{mL}$ and that this is a source of error in their experiment if they use this method.

\section{Preparation of the Dilute Bicarbonate Titrant Solution}

In each trial, one level teaspoon $(\sim 4.5 \mathrm{~g})$ of baking soda was measured into a $100 \mathrm{~mL}$ measuring cup. The measuring cup was then filled with distilled $\mathrm{H}_{2} \mathrm{O}$ until the final volume of the solution was $100 \mathrm{~mL}$ with a concentration of $\sim 0.54 \mathrm{M}$.

\section{Preparation of a Warm Water Bath}

For each trial, a $3 \mathrm{~L}$ saucepan was placed on a stove and the $500 \mathrm{~mL}$ measuring cup containing the $100 \mathrm{~mL}$ of dilute vinegar solution was placed into the saucepan. The saucepan was filled with $\mathrm{H}_{2} \mathrm{O}$, ensuring that the water did not overflow into the vinegar solution and until the water level in the saucepan reached just above the solution level in the $500 \mathrm{~mL}$ measuring cup. A thermometer was inserted into the saucepan and the temperature of the stove adjusted such that the water in the saucepan did not exceed $37^{\circ} \mathrm{C}$. This warm water bath was created in order to allow for homogenous, easy, and safe adjustment of the temperature of the vinegar solution as seen in Fig. 1B.

\section{Titration of Dilute Vinegar Solution with Dilute Baking Soda}

A $0.5 \mathrm{~mL}$ syringe was rinsed with the dilute baking soda solution, then filled with the baking soda solution as seen in Fig. 1C. Increments of $0.5 \mathrm{~mL}$ of the baking soda solution were 


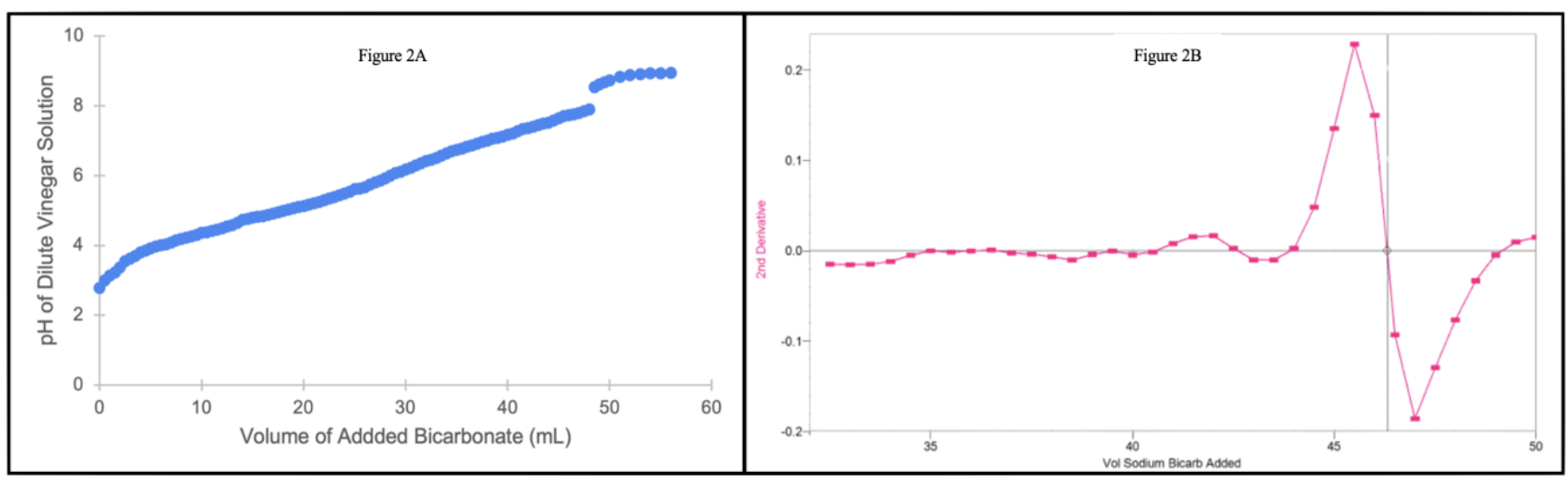

Figure 2 Acid-base Titration Results. A: Titration curve produced during Trial 3 of the titration of dilute vinegar with bicarbonate base at $37^{\circ} \mathrm{C}$. Based on the curve, the initial approximation of adding heat to the acetic acid-bicarbonate reaction is appropriate as the resulting curve simulates a weak acid-strong base titration with an endpoint $\mathrm{pH}>7.00$ and $\mathrm{a} \mathrm{pH} \approx \mathrm{pK}=4.76$ at the half-endpoint. $\mathbf{B}$ : Second derivative of the titration curve of titrating acetic acid with bicarbonate base at $37^{\circ} \mathrm{C}$. Based on the curve, the initial approximation of adding heat to the acetic acid-bicarbonate reaction to simulate weak acid-strong base chemistry is appropriate and accurate as there is only one endpoint that is clearly identifiable at $\sim 46.3 \mathrm{~mL}$ of bicarbonate base.

dropped into the dilute vinegar solution, ensuring that the solution properly mixed by swirling the vinegar solution after each addition of baking soda. The $\mathrm{pH}$ of the vinegar solution was measured using the calibrated Ruolan digital $\mathrm{pH}$ meter and recorded along with the volume after the addition of baking soda. The endpoint was determined as the point at which the $\mathrm{pH}$ of the vinegar solution made a large incremental increase. After this apparent endpoint, at least five additional increments of baking soda were added to complete the titration curve.

\section{Determining the Titration Endpoint and Error Analysis}

In each trial, a titration curve was generated by graphing the $\mathrm{pH}$ of the vinegar solution against the total volume of baking soda added. The true endpoint of the titration was determined graphically by plotting the second derivative of the function of $\mathrm{pH}$ versus volume of sodium bicarbonate added using Logger Pro (https://www.vernier.com/downloads/). The point at which the graph of the second derivative crossed the $\mathrm{x}$-axis during a recognizable spike pattern was determined to be the volume of sodium bicarbonate added at the equivalence point. ${ }^{21}$ The average value of the three trials was calculated and reported as the mean +/- the standard deviation. Using this average endpoint, the concentration of acetic acid in the original vinegar solution was calculated and compared to the advertised $6 \%$ acidity composition and the percent error was computed using Eq. 4-7.

\section{RESULTS AND DISCUSSION}

This experiment effectively demonstrated the basics of acid/base chemistry and the importance of accuracy and precision to students. Since the titration was completed using readily available household equipment, the precision of each student in measuring volumes was paramount as each household instrument was limited in its accuracy.
Table 1: Data collected during the three trials of acetic acid titration using bicarbonate base.

\begin{tabular}{l|l|l|l}
\hline Trial: & $\begin{array}{l}\text { Initial pH } \\
\text { of Dilute } \\
\text { Vinegar } \\
\text { Solution } \\
( \pm 0.01):\end{array}$ & $\begin{array}{l}\text { Final pH at } \\
\text { the } \\
\text { Endpoint } \\
( \pm 0.01):\end{array}$ & $\begin{array}{l}\text { Titration } \\
\text { Endpoint } \\
( \pm 0.25 \mathrm{~mL}):\end{array}$ \\
\hline 1 & 2.80 & 8.38 & 44.7 \\
2 & 2.88 & 6.30 & 46.3 \\
3 & 2.78 & 8.53 & 48.3 \\
\hline
\end{tabular}

Table 1 illustrates the endpoint values obtained during each trial of the titration. The average endpoint for the titration was determined to be $46.4 \mathrm{~mL}( \pm 0.43 \mathrm{~mL})$. Using the average endpoint, the percent composition of acetic acid in the original vinegar solution and the percent error from the advertised value was determined as follows, where $M$ represents concentrations in moles per liter and $V$ represents volumes in liters ${ }^{19}$ :

$$
\begin{aligned}
& \begin{array}{l}
M_{\text {Dilute Acetic Acid }} V_{\text {Dilute Acetic Acid }} \\
=
\end{array} \\
& M_{\text {Bicarbonate }} V_{\text {avg endpoint }}
\end{aligned}
$$

$$
\begin{aligned}
M_{\text {Whole Foods Vinegar }} & =M_{\text {Dilute Acetic Acid }} \times 5 \\
= & \frac{M_{\text {Bicarbonate }} V_{\text {avg endpoint }}}{V_{\text {Dilute Acetic Acid }}} \times 5
\end{aligned}
$$

Percent Error $=\frac{\left|M_{\text {Whole Foods Vinegar }}-1.0437 M\right|}{1.0437 M} \times 100$

Using the average endpoint, the concentration of acetic acid in the Whole Foods vinegar solution was determined to be $1.24 \mathrm{M}$, which results in a percent error of $19 \%$. Thus, the 
calculated acidity is 19\% higher in the Whole Foods vinegar solution than is advertised.

This elevated concentration of acetic acid might be the result of acidic impurities present in the white vinegar solution. During the fermentation process, for example, other organic acids are produced, including lactic acid, so the presence of these impurities can increase the volume of bicarbonate needed to titrate the solution. ${ }^{22}$ Although the presence of these additional acids might account for some of the elevation in the calculated concentration, the acidity of the vinegar solution is, regardless, higher than the reported $6 \%$ value.

Analysis of the titration curves produced during the experiment validates the approximation of using heat to simulate a weak acid-strong base titration through a weak acid-weak base titration of the acetic acid-bicarbonate system. ${ }^{1}$ Fig. 2A demonstrates the appropriateness of the approximation as the resulting titration curve closely resembles a weak acidstrong base titration with an endpoint $\mathrm{pH}$ that lies above 7.00 and a $\mathrm{pH}$ that approximates the $\mathrm{pK}_{\mathrm{a}}(=4.76)$ of acetic acid at the half-equivalence point, which is normally expected in a weak acid-strong base titration. ${ }^{19,20}$ Furthermore, Fig. 2B illustrates the accuracy of the at-home titration methodology since the graph of $\frac{d^{2}(p H)}{d V^{2}}$ indicates a clear, single endpoint at approximately $46 \mathrm{~mL}$, which is expected during a monoprotic weak acid-strong base titration. ${ }^{19}$

\section{Error Analysis of the At-Home Experiment}

Despite the success of this experiment in utilizing common athome instruments to examine the validity of the acetic acid concentration in Whole Foods white vinegar, the experiment is still subject to some error. Of note, much of the glassware and measuring instruments are limited in their accuracy. Even for calibrated instruments, such as the digital $\mathrm{pH}$ meter, accuracy is limited to $\pm 0.01 \mathrm{pH}$. Further, in titrations of weak acids, the determined endpoint is often slightly more basic than the true equivalence point owing to a larger volume of incremental base being added than the equivalence point in order to observe the $\mathrm{pH}$ change that occurs at the equivalence point. ${ }^{23}$ Although computer software was used to interpolate the value of the true equivalence point, this value is an estimation of the computer program and, therefore, error in the equivalence point is still present.

The largest source of error, however, stems from the amount of baking soda used. Due to two of the three students not having access to a digital mass balance, the student with the mass balance measured the mass of baking soda in one standard teaspoon as $4.5 \mathrm{~g}$. Thus, a teaspoon was used to measure baking soda during each trial by all students for consistency. Previous studies on household measuring instruments have cited the glassware as having varied accuracy even between repeat measures with the same instrument. ${ }^{24}$ Therefore, because of this possible mass error and the error associated with the accuracy of other at-home titration instruments, this titration may be subjected to a certain degree of uncertainty.

\section{Applicability of the At-home Experiment}

This experiment can be adapted to serve additional educational and further verification purposes. In terms of education, students may vary the initial dilution factor of the vinegar solution and exchange this diluted solution with a partner. Since the partner is blinded to the dilution factor, the student may use our at-home titration techniques to determine the concentration of the dilute vinegar solution and compare this concentration to the original vinegar concentration, thereby allowing the student to determine the dilution factor that was used. In terms of verification purposes, students may expand this experiment to include different brands of white vinegar and determine the accuracy of the advertised acetic acid concentration in these brands. The results may then be examined to determine the brand whose advertising is closest to the calculated concentration of acetic acid in the vinegar solutions.

The verification of cheap, easily accessible $\mathrm{pH}$ meters in at-home laboratories also illuminates opportunities for other future labs. For example, if one assumes that the concentration of commercially accessible vinegar is reported accurately, students could use it to standardize a solution of sodium bicarbonate. This sodium bicarbonate could then be used to titrate other household acids, such as lemon juice, to calculate the acid (citric acid, in this case) concentration. Another way to increase the educational value of labs using household products may be to encourage a teaching assistant or professor with access to a lab to record "known" values of variables including the mass, $\mathrm{pH}$, or other metrics of standard measurements of common household chemicals. An example of this might be the mass of a teaspoon of sodium bicarbonate, or the $\mathrm{pH}$ of a $5 \%$ commercial vinegar solution.

\section{CONCLUSIONS}

In summary, our experiment demonstrated a reliable and safe at-home experiment that utilizes university-level acid/base titration techniques to determine the acetic acid concentration in Whole Food white vinegar with sodium bicarbonate in the form of baking soda. The calculated concentration of the Whole Foods white vinegar is $19 \%$ higher than advertised. However, it is important to consider the limitations in accuracy and precision for at-home experiments. This experiment bridged the educational gap of distance learning by providing a practical, home-based application of theoretical acid/base chemistry learned in an online laboratory education. Our methodology can be readily adopted by online college and high school laboratory courses and included in course curriculum as a safe, accessible alternative to university acid/base experiments to supplement online laboratory education.

\section{AUTHOR INFORMATION}

\section{*Corresponding Author:}

*Ling Hao, PhD - Assistant Professor, Department of Chemistry, The George Washington University, Science and Engineering Hall, 800 22nd Street NW, Washington, D.C. 20052, USA; orcid.org/0000-0002-0106-5266;

Email: linghao@gwu.edu

\section{Author Contributions}

†These authors contributed equally as co-first author. 
The manuscript was written through contributions of all authors. All authors have given approval to the final version of the manuscript. Integrity of the data: P.P, L.B, M.G, and J.P had full access to all the data in the study and take responsibility for the integrity of the data. Concept and design: P.P, L.B, and M.G conceived the study concept and the study design was by P.P, L.B, and M.G. Acquisition, analysis, or interpretation of data: P.P, L.B, M.G, and J.P acquired and analyzed the data. Drafting of the manuscript: P.P, L.B, M.G, and J.P drafted the manuscript. Critical revision of the manuscript for important intellectual content: All co-authors provided critical revisions of the manuscript. Administrative, technical, or material support: LH provided guidance to PP, LB, MG, and JP. Study supervision: The study was conducted under the supervision of LH.

\section{Notes}

The authors declare no competing financial interest.

\section{ACKNOWLEDGEMENTS}

This project was supported by the Department of Chemistry at the George Washington University, through Chem 2123W course: Introductory Quantitative Analysis Laboratory. We also acknowledge Prof. LaKeisha M. McClary in the Chemistry Department for co-designing the syllabus of Chem $2123 \mathrm{~W}$. This virtual course was featured on the GW News: (https://gwtoday.gwu.edu/kitchen-chemistry-homeexperiments-enhance-remote-learning)

\section{REFERENCES}

1. Andrews JL, de Los Rios JP, Rayaluru M, et al. Experimenting with At-Home General Chemistry Laboratories During the COVID-19 Pandemic. J Chem Educ. doi:10.1021/acs.jchemed.0c00483

2. Garris CP, Fleck B. Student evaluations of transitionedonline courses during the COVID-19 pandemic. Scholarsh Teach Learn Psychol. Published online October 19, 2020. doi:10.1037/st10000229

3. Dandurand F, Shultz TR, Onishi KH. Comparing online and lab methods in a problem-solving experiment. Behav Res Methods. 2008;40(2):428-434 doi:10.3758/BRM.40.2.428

4. Schultz M, Callahan DL, Miltiadous A. Development and Use of Kitchen Chemistry Home Practical Activities during Unanticipated Campus Closures. J Chem Educ. 2020;97(9):2678-2684.

doi:10.1021/acs.jchemed.0c00620

5. Kelley EW. Reflections on Three Different High School Chemistry Lab Formats during COVID-19 Remote Learning. $J$ Chem Educ. 2020;97(9):2606-2616. doi:10.1021/acs.jchemed.0c00814

6. Johnston CS, Gaas CA. Vinegar: medicinal uses and antiglycemic effect. MedGenMed. 2006;8(2):61. http://www.ncbi.nlm.nih.gov/pubmed/16926800

7. "Acetic Acid." Epidemiology, Virginia Department of Health, Oct. 2018, www.vdh.virginia.gov/epidemiology/epidemiology- factsheets/acetic-acid/

8. Hansen LD, Fellingham GW, Russell DJ. Simultaneous determination of equilibrium constants and enthalpy changes by titration calorimetry: Methods, instruments, and uncertainties. Anal Biochem. 2011;409(2):220-229. doi:10.1016/j.ab.2010.11.002

9. ChemCollective - Determine the Concentration of Acetic Acid in Vinegar. Accessed June 16, 2021. http://chemcollective.org/activities/autograded/131

10. Johnston CS. Strategies for Healthy Weight Loss: From Vitamin C to the Glycemic Response. J Am Coll Nutr. 2005;24(3):158-165. doi:10.1080/07315724.2005.10719460

11. Chang J, Han SE, Paik SS, Kim YJ. Corrosive Esophageal Injury due to a Commercial Vinegar Beverage in an Adolescent. Clin Endosc. 2020;53(3):366-369. doi:10.5946/ce.2019.066

12. Bachow BH. Intravesical acetic acid in combination with prophylactic methenamine and ascorbic acid to decrease the incidence of recurrent urinary tract infections associated with intermittent urinary self-catherization: A case report. Urol Case Reports. 2021;35:101531. doi:10.1016/j.eucr.2020.101531

13. Quade BN, Parker MD, Occhipinti R. The therapeutic importance of acid-base balance. Biochem Pharmacol. 2021;183:114278. doi:10.1016/j.bcp.2020.114278

14. Wilkins JL. Challenges and Opportunities Created by the COVID-19 Pandemic. $J$ Nutr Educ Behav. 2020;52(7):669-670. doi:10.1016/j.jneb.2020.05.005

15. "Sodium Bicarbonate." National Center for Biotechnology Information. PubChem Compound Database, U.S. National Library of Medicine, pubchem.ncbi.nlm.nih.gov/compound/Sodium- bicarbonate.

16. Lanni LM. Filling a Plastic Bag with Carbon Dioxide: A Student-Designed Guided-Inquiry Lab for Advanced Placement and College Chemistry Courses. J Chem Educ. 2014;91(9):1390-1392. doi:10.1021/ed400901x

17. Antony E, Mitchell L, Nettenstrom L, Speckhard D. When A + B $\neq$ B + A. J Chem Educ. 2000;77(9):11801181.

18. Siqueira LA, Nunes IS, Almeida Junior PL, et al. Accurate automatic titration procedure for low sharpness and dichroism in end point detection using digital movies as detection technique. Microchem J. 2017;133:593-599. doi:10.1016/j.microc.2017.04.041

19. Harris DC, Lucy CA. Ch. 11: Acid-Base Titrations. In: Quantitative Chemical Analysis. 9th ed. Freeman Custom Publishing; 2016:233-257.

20. "Acetic Acid." National Center for Biotechnology Information. PubChem Compound Database, U.S. National Library of Medicine, pubchem.ncbi.nlm.nih.gov/compound/Aceticacid\#section=Solubility.

21. Zellmer DL. The Derivative End Point Methods. zimmer.csufresno.edu/ davidz/Chem102/Derivative/Derivati ve.html

22. Maicas S. The Role of Yeasts in Fermentation Processes. Microorganisms. $2020 ; 8(8): 1142$ doi:10.3390/microorganisms 8081142

23. Harris DC, Lucy CA. Ch. 7: Let the Titrations Begin. In: Quantitative Chemical Analysis. 9th ed. Freeman Custom Publishing; 2016:145-147.

24. Coe JB, Rankovic A, Edwards TR, Parr JM. Dog owner's accuracy measuring different volumes of dry dog food using three different measuring devices. Vet Rec. 2019;185(19):599-599. doi:10.1136/vr.105319. 